\title{
A 64-channel, 1.1-pA-accurate On-chip Potentiostat for Parallel Electrochemical Monitoring
}

\author{
Peishuo Li*, Tom R. Molderez*, Frederik Ceyssens*, Korneel Rabaey ${ }^{\dagger}$ and Marian Verhelst* \\ *ESAT-MICAS, KU Leuven, Kasteelpark Arenberg 10, B-3001 Leuven, Belgium \\ ${ }^{\dagger}$ CMET, UGent, Coupure Links 653, B-9000 Gent, Belgium \\ Email: \{peishuo.li, tom.molderez, frederik.ceyssens, marian.verhelst\}@kuleuven.be, korneel.rabaey@ugent.be
}

\begin{abstract}
Electrochemical monitoring is crucial for both industrial applications, such as microbial electrolysis and corrosion monitoring as well as consumer applications such as personal health monitoring. Yet, state-of-the-art integrated potentiostat monitoring devices have few parallel channels with limited flexibility due to their channel architecture. This work presents a novel, widely scalable channel architecture using a switch capacitor based Howland current pump and a digital potential controller. An integrated, 64-channel CMOS potentiostat array has been fabricated. Each individual channel has a dynamic current range of $120 \mathrm{~dB}$ with $1.1 \mathrm{pA}$ precision with up to $100 \mathrm{kHz}$ bandwidth. The on-chip working electrodes are post-processed with gold to ensure (bio)electrochemical compatibility.
\end{abstract}

\section{INTRODUCTION}

Parallel, accurate electrochemical monitoring is crucial for high-throughput and/or widely variable applications in the industrial and consumer fields. To study individual electrochemical half reactions, a potentiostat architecture with a 3electrode setup is used [1]. For the development of novel microbial electrochemical reactors, multichannel potentiostats allow to tune the electrical settings optimally with flexible stimulation patterns, irrespective of the natural statistical variation of the microorganisms or the environment dynamics [2]. Similarly, for the study of novel anti-corrosion coatings, a very slow aging process, parallelization notably speeds up those experiments [3]. For personal healthcare monitoring, various biomolecules act as electrochemical transducers. As such, a multichannel on-chip potentiostat can assess the impact of different chemicals simultaneously [4] at low cost.

Current SotA parallel on-chip potentiostats focus mostly on parallelizing the readout circuitry, by measuring the current of each individual channel while keeping the potential equal for all channels. As such only a single, analog potential controller is required. In [5], an active microelectrode array (MEA) for DNA analysis is presented with 16 parallel readout channels. Using a more advanced circuit architecture, [6] expanded the number of parallel readout channels to 1024 with $93 \mathrm{~dB}$ dynamic range. Yet, both have only a single stimulation unit. While this is sufficient for studying the spatial effects of electrochemical reactions, a truly parallel stimulation and sensing potentiostat with large dynamic range is required for monitoring those high-throughput and/or widely variable processes.

In [7], a multi-functional MEA is presented with 59760 electrodes and 2048 parallel readout channels. The number

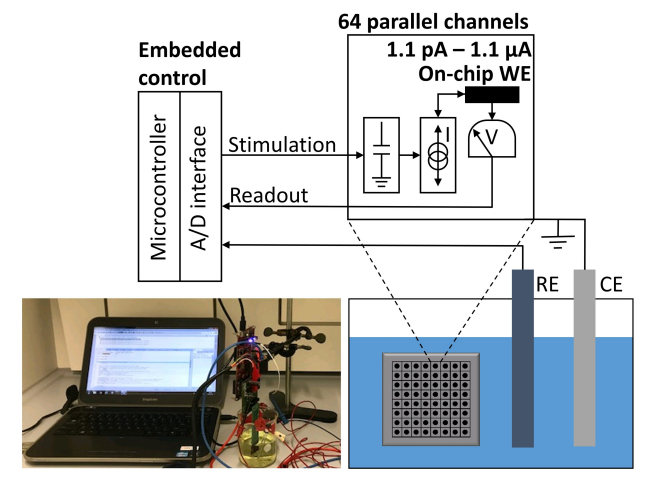

Fig. 1. System overview

of independent stimulation units is expanded to 16. In [8] the multiplexed cyclic voltammetry with 16 parallel independent stimulation and sensing potentiostats is further investigated.

Further expanding the amount of independent potentiostats operating simultaneously is however challenging for the SotA architectures due to the limited chip input/output pin numbers. Moreover, the largest dynamic range of the current sensing unit in SotA designs is below $100 \mathrm{~dB}$. Therefore, in this paper a current stimulation structure is used with a boosted dynamic range. The potentiostat control is maintained by the digital feedback loop running on an external microcontroller. The lift-off process used to pattern electrodes on chip can be modified in order to improve the process yield. Compared to the SotA designs, this paper therefore introduces the following novelties: 1) a scalable multiplexing architecture resulting in 64 independent stimulation channels with only a single input pin and a single output pin, and fully software-programmable control with an arbitrary waveform; 2) a switch capacitor based current pump with bipolar output, $120 \mathrm{~dB}$ wide dynamic range, and $1.1 \mathrm{pA}$ resolution; 3) a post-processing technique with thick metal and an extra protection layer to pattern on-chip working electrodes (WEs).

This paper is structured as follows: First, section II introduces the channel architecture. The circuit implementation is discussed in section III. Next, section IV details the postprocessing steps to pattern the electrodes. Finally, section $\mathrm{V}$ presents the electrical measurement and chemical cyclic voltammetry results. 


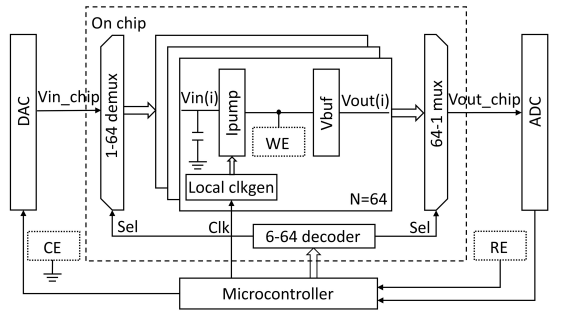

Fig. 2. Channel multiplexing architecture

\section{SYSTEM ARCHITECTURE}

The 64-channel potentiostat consists of three parts: the multiplexing architecture, the bipolar current-stimulation/voltagesensing potentiostat channel and the on-chip working electrode, shown in Fig. 1. They are discussed below.

\section{A. Multiplexing architecture}

A flexible, scalable multiplexing architecture is shown in Fig. 2. A digital controller is used to close the feedback loop. Only one input/output pair together with a single external $\mathrm{ADC} / \mathrm{DAC}$ is required to control all channels using timedivision multiplexing.

Through the selection signal Sel, driving the 1-64 multiplexer and 64-1 demultiplexer, each channel can be selected individually. As such, the ADC can sense the output voltage of each channel, and send it to an off-chip, low-cost microcontroller (TI TMS320F28379D). The microcontroller runs a digital proportional-integral (PI) controller to maintain the output potential of each channel equal to a set-point by stimulating the channel's current. The set-point potential can be time-varying, represented by a fully-programmable waveform.

A capacitor at the input of every channel stores the channelspecific current-stimulation input signal when the channel is not selected. In this way, all 64 channels are stimulated simultaneously with only one input pin. The interface between the digital controller and the analog chip is composed of a single 12-bit, 250-kS/s ADC and DAC. This scheme is easily scalable to a large number of channels by increasing either the storage capacitor size or the mixed-signal sample rate.

\section{B. Potentiostat channel}

This work applies a current stimulation, voltage measurement architecture. The channel schematic is shown in Fig. 3. A Howland current pump is implemented with a switch capacitor topology. Such scheme has the benefit of a large dynamic current range, because the value of the equivalent resistor of a switch capacitor is inversely proportional to the tunable switch clock frequency. All channels share the same switch capacitor clock to set the coarse output range. The input voltage of the current pump is channel-specific and used for output fine-tuning.

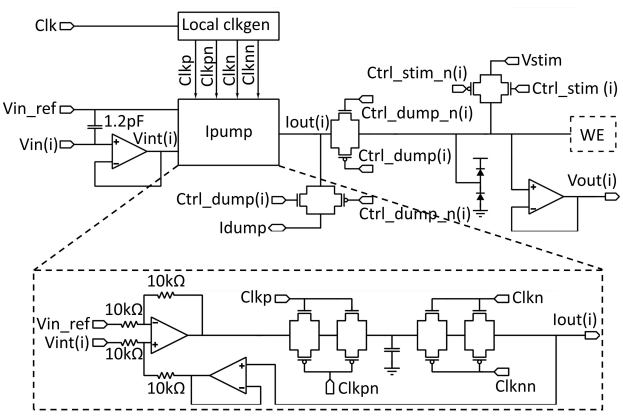

Fig. 3. Schematic of a single channel

\section{On-chip working electrode}

The working electrodes are integrated on chip to allow a wide parallelization. The top metal layer of the non-processed chip is aluminum (Al). As $\mathrm{Al}$ is not electrochemically stable, each electrode receives a biocompatible gold $(\mathrm{Au})$ coating during post-processing.

\section{CiRcuit LEVEL DESIGN}

The chip is designed in $180 \mathrm{~nm}$ technology with $3.3 \mathrm{~V}$ supply. Each channel consists of a memory capacitor, an output voltage buffer (Vbuf) and a bipolar current pump (Ipump).

The memory capacitance value of $1.2 \mathrm{pF}$ limits the voltage drop to be less than $1 \mathrm{mV}$ when cycling through all 64 channels while have no impact on the required bandwidth.

In order to run a complete cyclic voltammetry with sufficient accuracy, the targeted voltage sensing swing is set to $2 \mathrm{~V}$ and the targeted resolution is $1 \mathrm{mV}$. The output voltage measurement unit is implemented with an opamp in unity feedback. A rail-to-rail configuration [9] is adopted to maximize the swing without adding much extra area.

A modified Howland current pump is used to provide the wide-dynamic bipolar currents [10]. The current pump uses two opamps, four $10 \mathrm{k} \Omega$ resistors and one tunable resistor $\left(R_{-}\right.$tune $)$, generating a bipolar current Iout $=\left(\right.$ Vin $\left.-V i n \_r e f\right) / R_{-}$tune. The first opamp together with all the resistors generates a differential bipolar current while the second opamp isolates the output load from the internal resistors.

The tunable resistor $\left(R_{-}\right.$tune $)$is implemented with a switch capacitor $(R=1 / f C)$, which results in a large dynamic range by adjusting the switch clock frequency. For the switch capacitor, a $100 \mathrm{fF}$ small capacitor $C$ is chosen to achieve an extremely large equivalent resistor and as such a very low minimal current resolution. The switches are implemented as transmission gates to maximize the output swing. The switch size is minimized to reduce the clock feedthrough which decreases the output accuracy. Additional switches are added in series to decrease the static leakage. Non-overlapping clocks are locally generated to prevent leakage during switching.

During simulation, the output resistance of the current pump is about 20 times larger than $R \_$tune, leading to a wide load range. $10 \%$ accuracy can be achieved at 500fA output current 


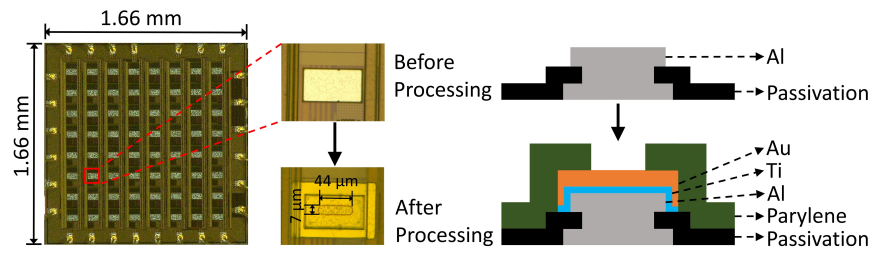

Fig. 4. Chip die photograph and post-processing

(50mV input, $100 \mathrm{~Hz}$ clock frequency). The thermal noise is not an issue since the current pump bandwidth is coupled to the switch clock frequency and shrinks in proportion with the current range.

To decouple the current pump from the memory capacitor, an input voltage buffer stage is added. For testing purposes, additional transmission gates are inserted between the current pump and the voltage buffer.

\section{POST-PROCESSING}

The biocompatible interface between the chip and a (bio)chemical solution is achieved by patterning $\mathrm{Au}$ on top of each $\mathrm{Al}$ electrode. It was found that the surface of the $\mathrm{Al}$ bond pad is not sufficiently flat, especially at the edges. A process is designed to overcome this issue, which consists of a) gluing the diced chip on a supporting wafer, together with surrounding dummy chips; b) applying and patterning a two-layer liftoff resist stack; c) sputtering $100 \mathrm{~nm} \mathrm{Ti}$ as thick diffusion barrier and then a thick Au layer $(400 \mathrm{~nm})$; d) dissolving the liftoff resist; e) depositing $5 \mu \mathrm{m}$ of Parylene C; f) patterning the Parylene by lithography and oxygen plasma. Photoresist exposure was done using direct laser writing.

An electrode after post-processing is shown in Fig. 4. The extra Parylene step provides a protection on the $\mathrm{Al}$ electrode edges. To further prevent possible $\mathrm{Al}$ corrosion at the edge after Au deposition, an edge-shifted pattern is used: The Ti/Au layer is patterned with $15 \mu \mathrm{m}$ extra margin at each side on top of the Al electrode, while the Parylene opening shrinks with $35 \mu \mathrm{m}$ at each side on top of the Ti/Au pattern. The final WE area is $7 \times 44 \mu \mathrm{m}^{2}$.

With the improved process, not all $\mathrm{Al}$ electrodes are completely covered with Au due to the bond pad surface topography. New processes are being investigated to completely solve this issue.

\section{Measurement Results}

The chip is first measured electrically before postprocessing, and then electrochemically after post-processing.

\section{A. Electrical measurements}

1) Voltage sensing: The DC output error of the voltage buffer is measured and calibrated through a two-point calibration: From two consecutive measurement points, a 2coefficient fitting (gain and offset compensation) is done to calculate the voltage buffer input corresponding to the expected output. Then the output is measured again with compensated

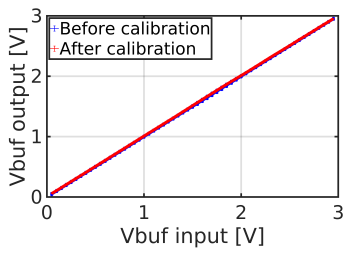

(a)

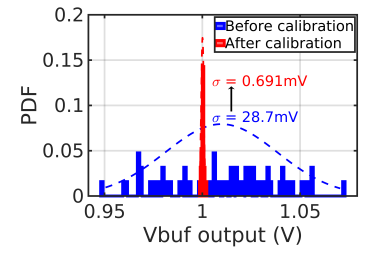

(b)
Fig. 5. Vbuf DC output (a) and distribution at $1 \mathrm{~V}$ input (b) before and after calibration

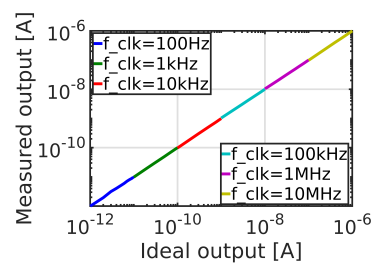

(a)

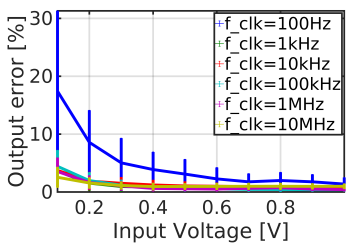

(b)
Fig. 6. Ipump DC output (a) and error after calibration (b)

input and compared with the expected value. Fig. 5 shows the voltage buffer output before and after calibration.

2) Current stimulation: The current pump DC output is measured through the voltage across an external resistor (from $100 \mathrm{k} \Omega$ to $1 \mathrm{G} \Omega$ ). The wide range from $1 \mathrm{pA}$ to $1 \mu \mathrm{A}$ for one channel is shown in Fig. 6a, with maximum $0.35 \%$ nonlinearity. The output current is configurable by both the clock frequency and the input voltage.

A similar calibration procedure is used to calibrate all 64 channels. The post-calibration error is below $10 \%$ except in the pA range output $(100 \mathrm{~Hz}$ clock frequency), where the maximum post-calibration error equals $31 \%$, as shown in Fig. $6 \mathrm{~b}$. This error is much larger than simulation $(<1 \%)$, probably caused by the insufficient measurement setup isolation. Note that only the positive current output is shown, as similar measurements were obtained for the negative current.

The output resolution with the current measurement set-up is evaluated from the measured noise over the system time constant $(\sim 1 \mathrm{~s}$ for $100 \mathrm{~Hz}$ clock), resulting in $1.14 \mathrm{pA}$. The $1.1 \mu \mathrm{A}$ measured maximum current is limited by the maximum allowed switch-capacitor clock frequency, set by the opamp bandwidth.

3) Closed loop system: The system bandwidth needs to be sufficiently large in order to run dynamic measurements. The transfer function of the system is measured with a dummy load resistor. The current pump input and the voltage buffer output are connected to the network analyzer. The external microcontroller is not taken into account because it operates at a fast clock $(200 \mathrm{MHz})$. The results with different clock frequencies are shown in Fig. $7.117 \mathrm{kHz}$ bandwidth is achieved with $10 \mathrm{MHz}$ clock frequency and $1 \mathrm{~Hz}$ bandwidth can be extrapolated for the $100 \mathrm{~Hz}$ clock or $1 \sim 10 \mathrm{pA}$ operation. However, the measured bandwidth is actually limited by the load resistor and it is not possible to use smaller load resistors 
TABLE I

STATE-OF-THE-ART COMPARISON

\begin{tabular}{|c|c|c|c|c|c|}
\hline Reference & JSSC-08 [5] & JSSC-17 [7] & TCAS-18 [8] & ISSCC-19 [6] & This work \\
\hline CMOS fabrication technology $[\mu \mathrm{m}]$ & 0.25 & 0.18 & 0.35 & 0.25 & 0.18 \\
\hline Chip area $\left[\mathrm{mm}^{2}\right]$ & $5 \times 3$ & $10.1 \times 7.6$ & $3.79 \times 3.79$ & $7 \times 9$ & $1.66 \times 1.66$ \\
\hline $\mathrm{WE} \operatorname{size}\left[\mu \mathrm{m}^{2}\right]$ & $70 \times 70 \sim 100 \times 100$ & $3 \times 7.5$ & $20 \times 20$ & $\sim 30 \times 30$ & $7 \times 44$ \\
\hline Number of WE & 16 & 59760 & 256 & 1024 & 64 \\
\hline Number of independent channels & 1 & 16 & 16 & 1 & 64 \\
\hline $\operatorname{Imax}[\mu \mathrm{A}] /$ Sensitivity $[\mathrm{pA}]$ & $0.11 / 240$ & $\sim 1 / 1000$ & $13 / 1390$ & $0.0125 / 0.28$ & $1.1 / 1.1^{1}$ \\
\hline Current dynamic range[dB] & 53.2 & $\sim 60$ & 82.6 & 93 & 120 \\
\hline Measured voltage swing[V] & 1.25 & 1.0 & 5.2 & 1.5 & 2.5 \\
\hline Bandwidth $[\mathrm{Hz}]$ & $10 \mathrm{k}$ & $10 \mathrm{k}$ & $150 \mathrm{k}$ & 50 & $\begin{array}{r}\text { 117k@ } 0.1 \sim 1 \mu \mathrm{A} \\
\sim 1 @ 1 \sim 10 \mathrm{pA} \\
\end{array}$ \\
\hline Max. Power consumption[mW] & $\sim 10$ & 86 & 125 & $\sim 256$ & 125 \\
\hline
\end{tabular}

${ }^{1} 0.5$ in simulation

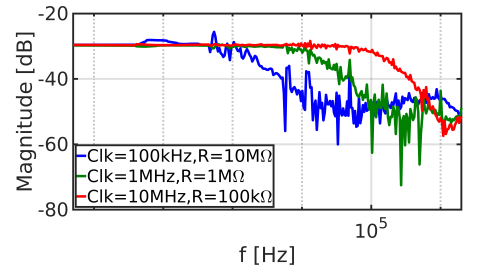

Fig. 7. System transfer function magnitude

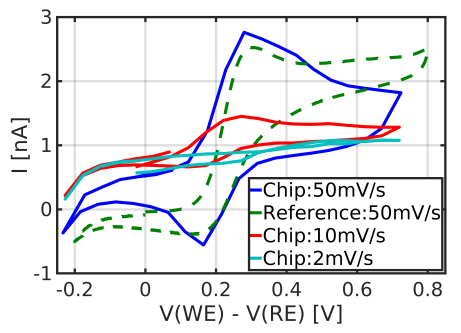

Fig. 8. Cyclic voltammetry results benchmarked with commercial potentiostat

due to the network analyzer dynamic range limitation.

\section{B. Electrochemical measurements}

Cyclic voltammetry (CV) in chemical solutions is used to verify the close loop performance and quality of the postprocessing. The bonded chip was emerged into $0.3 \mathrm{mmol} / \mathrm{L}$ potassium ferrocyanide solution with a commercial $\mathrm{Ag} / \mathrm{AgCl}$ RE (ALS, Japan) and a Pt wire as the CE. A closed loop CV was run at different scan rates, shown in Fig. 8. The result at $50 \mathrm{mV} / \mathrm{s}$ is compared with a commercial potentiostat (Gamry Reference $600+$ ) using a $100 \times 100 \mu \mathrm{m}^{2}$ dummy electrode array, normalized to the chip electrode area. The most likely cause of the observed differences are the boundary effects because of the smaller on-chip electrode.

\section{CONCLUSION}

This work presents a $180 \mathrm{~nm}$ CMOS 64-channel potentiostat. Compared to SotA (Table I), $4 \times$ more independent electrochemical characterization channels and $30 \mathrm{~dB}$ larger current dynamic range are achieved. These innovations make the chip feasible to monitor high-throughput, widely-variable electrochemical processes, such as microbial electrolysis, anticorrosion studies and biomolecule detection, with fast and flexible characterization. Further research on the post-processing is needed to improve the reliability.

\section{ACKNOWLEDGMENT}

The authors would like to thank the Hercules Foundation for heavy equipment (AKUL 034 and ZW1115) and FWO Vlaanderen for the research funding.

\section{REFERENCES}

[1] A. J. Bard et al., Electrochemical Methods: Fundamentals and Applications, 2nd Edition. Wiley, 2001.

[2] T. R. Molderez et al., "A current-driven six-channel potentiostat for rapid performance characterization of microbial electrolysis cells," IEEE Transactions on Instrumentation and Measurement, Mar. 2019.

[3] V. N. Rafla et al., "Coupled multi-electrode array with a sintered $\mathrm{Ag} / \mathrm{AgCl}$ counter/reference electrode to investigate AA7050-T7451 and type 316 stainless steel galvanic couple under atmospheric conditions," Journal of The Electrochemical Society, vol. 165, no. 9, pp. C562-C572, Jun. 2018.

[4] D. L. Bellin et al., "Electrochemical camera chip for simultaneous imaging of multiple metabolites in biofilms," Nature communications, vol. 7, no. 10535, Jan. 2016.

[5] P. M. Levine et al., "Active CMOS sensor array for electrochemical biomolecular detection," IEEE Journal of Solid-State Circuits, vol. 43, no. 8, pp. 1859-1871, Aug. 2008.

[6] A. Manickam et al., "A CMOS biosensor array with 1024 3-electrode voltammetry pixels and 93dB dynamic range," in 2019 IEEE International Solid-State Circuits Conference-(ISSCC), Feb. 2019, pp. 192-194.

[7] J. Dragas et al., "In vitro multi-functional microelectrode array featuring 59760 electrodes, 2048 electrophysiology channels, stimulation, impedance measurement, and neurotransmitter detection channels," IEEE Journal of Solid-State Circuits, vol. 52, no. 6, pp. 1576-1590, Jun. 2017.

[8] C. Giagkoulovits et al., "A 16 x 16 CMOS amperometric microelectrode array for simultaneous electrochemical measurements," IEEE Transactions on Circuits and Systems I: Regular Papers, vol. 65, no. 9, pp. 2821-2831, Sep. 2018.

[9] R. Hogervorst et al., "A compact power-efficient $3 \mathrm{~V}$ CMOS rail-to-rail input/output operational amplifier for VLSI cell libraries," IEEE Journal of Solid-State Circuits, vol. 29, no. 12, pp. 1505-1513, Dec. 1994.

[10] "A comprehensive study of the Howland current pump," Texas Instruments, Dallas, TX, USA, Tech. Rep. AN-1515, Apr. 2013. 